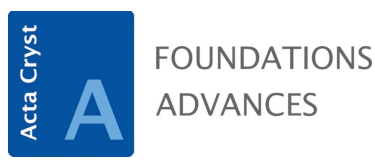

ISSN 2053-2733

Received 21 April 2020

Accepted 3 August 2020

Edited by U. Grimm, The Open University, United Kingdom

Keywords: weaves; knots; links; catenanes.

Supporting information: this article has supporting information at journals.iucr.org/a
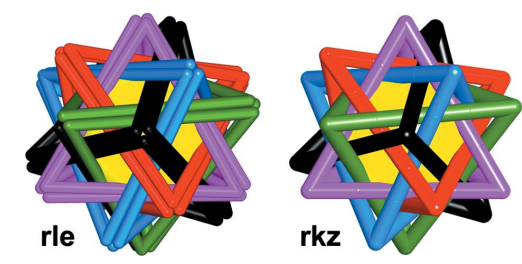

C 2020 International Union of Crystallography

\section{Isogonal weavings on the sphere: knots, links, polycatenanes}

\author{
Michael $\mathrm{O}^{\prime}$ Keeffe $^{\mathrm{a}}$ and Michael M. J. Treacy ${ }^{\mathrm{b} *}$ \\ ${ }^{\mathbf{a} S}$ School of Molecular Sciences, Arizona State University, Tempe, Arizona 85287, USA, and ${ }^{\mathbf{b}}$ Department of Physics, \\ Arizona State University, Tempe, Arizona 85287, USA. *Correspondence e-mail: treacy@asu.edu
}

Mathematical knots and links are described as piecewise linear - straight, nonintersecting sticks meeting at corners. Isogonal structures have all corners related by symmetry ('vertex'-transitive). Corner- and stick-transitive structures are termed regular. No regular knots are found. Regular links are cubic or icosahedral and a complete account of these (36 in number) is given, including optimal (thickest-stick) embeddings. Stick 2-transitive isogonal structures are again cubic and icosahedral and also encompass the infinite family of torus knots and links. The major types of these structures are identified and reported with optimal embeddings. The relevance of this work to materials chemistry and biochemistry is noted.

\section{Introduction}

\subsection{Knots and links}

Weavings, knots, links and related geometrical structures are currently of considerable interest in materials chemistry and biochemistry, as testified in recent reviews (Lim \& Jackson, 2015; Flapan, 2015; Bruns \& Stoddart, 2016; Pieters et al., 2016; Horner et al., 2016; Fielden et al., 2017) and in other recent reports (Danon et al., 2017; Wu et al., 2017; Kim et al., 2018; Zhang et al., 2018; Leigh et al., 2019; Sawada, Saito et al., 2019; Sawada, Inomata et al., 2019; Inomata et al., 2020). Suggested knots most suitable for self-assembly have recently been identified (Marenda et al., 2018). We are particularly interested in such structures from the perspective of reticular chemistry, which is concerned with the directed assembly of symmetrical frameworks from molecular components (Yaghi et al., 2003). Target materials include finite structures such as polyhedra (Tranchemontagne et al., 2008). Pursuant to that goal we analyze geometric structures as being piecewise linear, considering this as most relevant to chemical structures at the molecular level (Liu et al., 2018; O'Keeffe \& Treacy, 2020). In this approach we term the linear segments sticks, in accordance with established practice in knot theory and to emphasize that we are interested in structures with linear, non-intersecting, edges. In knots, threads and loops, sticks meet at 2-coordinated (2-c) vertices termed corners. For such structures a transitivity $c s t$ indicates that there are $c$ kinds (i.e. symmetry-related - 'one orbit') of corner, $s$ kinds of stick and $t$ kinds of thread or loop. In the jargon, corner- (vertex-) transitive structures are isogonal. Structures with transitivity 111 we term regular.

A major tenet of reticular chemistry is the principle of minimal transitivity, which holds that structures most likely to occur, and hence good targets for synthesis by design, are those with minimal transitivity (Li et al., 2014). In earlier work we surveyed the field with emphasis on periodic structures 
(Liu et al., 2018) and gave a detailed analysis of regular 2-periodic structures (O'Keeffe \& Treacy, 2020).

In the present work we focus on finite structures such as knots and links (chemically, polycatenanes) giving what we believe is a reasonably complete survey of isogonal structures. These are either stick-transitive (transitivity 1111 ), for which we believe all structures are identified, or stick 2-transitive (transitivity 121 ), a category that includes the infinite family of torus knots and links. Key structures are identified by a three-letter symbol and can be found in the Reticular Chemistry Structure Resource (RCSR, O'Keeffe et al., 2008) in the section on Polyhedra, keyword 'weaving'. We note an earlier study ( $\mathrm{Hu}$ et al., 2009) enumerating cubic and icosahedral polycatenane structures. These were derived by replacing faces of regular polyhedra with polygons with entwined edges. As discussed in the supporting information, these were not isogonal but had two or more kinds of corner (vertex).

A good account of knot theory, for those unfamiliar with the topic, is the book by Adams (1994). Knots and links have a characteristic crossing number, which may be informally defined as the minimal possible number of crossings in a planar drawing of the structure. Knots with a small number of crossings are identified by the Alexander-Briggs (AB) symbol $n_{i}$ in which $n$ is the number of crossings and $i$ is a serial number. Similarly, links have a symbol $n_{i}^{m}$ where $n$ is again the number of crossings, $m$ the number of linked loops and $i$ again is a serial number. A useful source of illustrations and information about individual knots with small crossing numbers is the Knot Atlas (http://katlas.org/wiki/Main_Page). However, we note that the number of knots increases rapidly with crossing number - there are $>10^{6}$ with crossing number $\leq 16$ (Hoste et al., 1998).

Traditionally, knots are analyzed and listed in order of increasing crossing number. However, in this work we find regular structures with crossing numbers of hundreds for which the methods of knot theory become inappropriate. We recall that simple crystalline materials have been made in which rings are catenated to hundreds of others (Bonneau \& O'Keeffe, 2015).

We find that finite stick-transitive structures are cubic or icosahedral and we give a full account of them. However, there is a large number of the corresponding stick 2-transitive cubic and icosahedral structures. We give data for all of these but restrict detailed descriptions to those that can be made with relatively thick sticks (defined below as large girth).

The other stick 2-transitive structures we recognize are embeddings of the well documented torus knots and links (Adams, 1994). We give data for these using our stick-corner approach.

We do not claim to have determined the symmetries of knots and links. A polyhedron (sensu stricto with a planar 3-connected graph) has a well defined maximum symmetry and all embeddings must be with that symmetry, or be a subgroup. Not so for knots. A well documented case is that of knot $4_{1}$ (Flapan, 1987, 1988). This knot is commonly illustrated as chiral, with symmetry $222\left(D_{2}\right)$, but it has also an achiral embedding with symmetry $\overline{4}\left(S_{4}\right)$ (see illustration in the supporting material). Curiously, these two symmetry groups have no group-subgroup relationship; indeed, both have order 4 , so the question of the symmetry of the knot is moot. But the knot is achiral; it can readily be shown that the chiral representation is isotopic with its mirror image (Fielden et al., 2017).

At the time this paper was submitted for publication we were unaware of the book Orderly Tangles (Holder, 1983). This work contains numerous illustrations of piecewise linear models of knots and links. Particularly relevant to our work is the part on 'regular polylinks' which contains illustrations of many of the regular structures described below (Section 2). Holden's work was further elaborated by Lang (2002) who enumerated the possibilities but gave no other details. We find the same number of regular structures and present details. We believe that illustrations, specific coordinates and catenation details of all 36 structures are presented here for the first time. We remark also that we could find no reference to those books in the physical science literature.

\subsection{Weavings on the sphere}

Weavings on the sphere can conveniently be derived from polyhedra with 4-c vertices (Jablan et al., 2011; Thompson \& Hyde, 2018). A weave commonly found in artwork and models is related to the triaxial kagome weave, kgm, shown in Fig. 1. Starting from the simple tiling of the plane by hexagons, hcb (top left), the 4-c tiling, $\mathbf{~ k g m ~ ( c e n t r e ~ t o p ) , ~ i s ~ d e r i v e d ~ a s ~ t h e ~ e d g e ~}$ net obtained by placing vertices (corners) in the midpoints of the edges of the original hcb net, and then connecting the new vertices (O'Keeffe et al., 2008). This kagome pattern in turn leads to the kagome weave, kgm-w (top right). Replacing 12 hexagons by pentagons will produce a simple (3-c) tiling of the sphere, deg (12 pentagons, 30 hexagons), from which a triaxial weaving can, in turn, be derived - that is, deg-e-w from deg-e (middle row of Fig. 1). It can be seen that the threads in deg-e-w are now closed, woven loops. Note that, since the
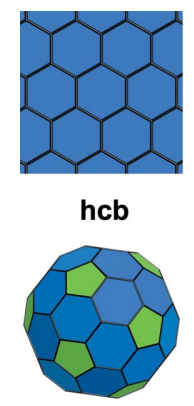

deg

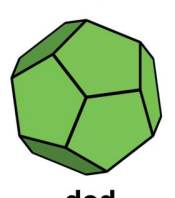

dod

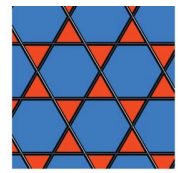

$\operatorname{kgm}(=$ hcb-e)
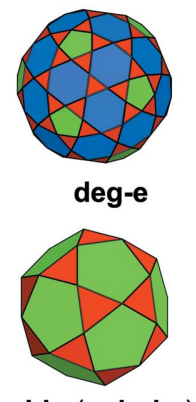

ido (= dod-e)

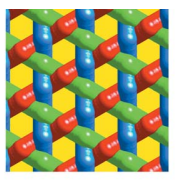

kgm-w
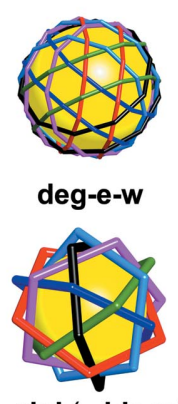

Figure 1

The derivation of spherical weaves from polyhedra. Left column shows the basic net; centre column shows the augmented net, generated by replacing each vertex with three; the right column shows the resulting weave when links are replaced with non-intersecting threads. 
surface of the polyhedron is non-planar, weaves can be derived directly by replacing polyhedron edges by sticks joining further neighbors. In the limit of an all-pentagon polyhedron (dod in the figure, lower right) it may be seen that the loops in rkd ( $\equiv$ dod-e-w $\equiv$ ido-w) are now regular pentagons and the structure is now regular (transitivity 111 ). In this communication we derive systematically all possible regular weavings on the sphere.

It might be noted that we talk about weaving on the sphere, but really the weaving is under-and-over (about) a surface just as weavings 'on the plane' are really under and over. Periodic weavings about the plane are three-dimensional even though they lack the $[00 t]$ generator for periodicity out of the plane, and exhibit one of the 80 layer-group symmetries (as opposed to one of the 17 plane-group symmetries). We mention this three-dimensionality because some of our structures are the torus knots and links. They are equally spherical weavings, but have the property that they can be drawn on the surface of a torus with non-intersecting curved threads. We show that these all have isogonal piecewise linear embeddings.

\subsection{Maximum-girth structures}

We define girth of a piecewise linear structure as the ratio of the shortest distance between sticks to the length of the longest stick (Liu et al., 2018; O'Keeffe \& Treacy, 2020). This defines the thickest stick that could be used to make a model of the structure and is similar to the procedure of finding the maximum-diameter rope that can be used to make structures from flexible constant-diameter ropes (Stasiak et al., 1998).

If coordinate space is systematically explored for a given pattern of linking, local points of maximum girth can be identified. These generally correspond to topologically distinct structures but, as noted in earlier work, occasionally two local maxima may correspond to isotopes (O'Keeffe \& Treacy, 2020). Usually, these can be detected by inspection but, in the case of stick 2-transitive icosahedral structures, their complexity (crossing numbers in the hundreds) precludes easy determination. On the other hand, the results are generally unambiguous, particularly for regular structures.

\section{Regular (stick-transitive) structures}

The stick-transitive (i.e. transitivity $c s t=111$ = 'regular') spherical weavings are mathematical links, in chemistry polycatenanes, with cubic or icosahedral symmetry. As explained below, the loops are planar regular polygons linked to other such polygons by simple 2-crossing (Hopf) links. A structure with $n$ loops is known as an $[n]$ catenane. We use the catenation symbol $u-v-w$ to describe a regular structure of $u$ loops that are $v$-gons, each of which is linked to $w$ others.

It should be clear that catenated planar loops are only possible with symmetries lacking mirror planes. They must have sticks that are all related by symmetry axes of order 3 or greater and which make a finite regular group. These axes cannot be parallel. This limits the possible symmetries to the
Table 1

Data for the regular, stick-transitive, structures $(c=1, s=1, t=1)$.

The coordinates are Cartesian and are normalized such that the stick length equals 1 . Sticks are defined as connections between vertex $x, y, z$ to: $-z,-x, y$ for triangles in groups 23 and 432; $z, x, y$ for triangles in group 235; $x, z,-y$ for squares; the matrix operation $\left[\frac{1}{2}, g,-G ; g, G, \frac{1}{2} ; G,-\frac{1}{2}, g\right](x, y, z)$ for pentagons (in rkd-rkn), where $G=(\sqrt{5}+1) / 4, g=(\sqrt{5}-1) / 4$.

\begin{tabular}{|c|c|c|c|c|c|c|}
\hline Catenation & Symbol & Group & Girth & $x$ & $y$ & $z$ \\
\hline $4-3-3$ & rka & 432 & 0.1380 & 0 & $1 / \sqrt{6}$ & $1 / \sqrt{6}$ \\
\hline $4-3-3$ & rkh & 23 & 0.0893 & 0.2271 & 0.6715 & 0.0248 \\
\hline $6-4-4$ & rkb & 432 & 0.1408 & 0.2041 & 0.6487 & -0.2813 \\
\hline $6-4-4$ & rkc & 432 & 0.0826 & 0.5078 & 0.6790 & -0.1975 \\
\hline $8-3-3$ & rki & 432 & 0.0938 & 0.1934 & 0.6145 & 0.3126 \\
\hline $8-3-3$ & rkj & 432 & 0.0606 & 0.0160 & -0.2076 & 0.5756 \\
\hline $8-3-3$ & rkk & 432 & 0.0949 & 0.4173 & 0.3970 & -0.0617 \\
\hline $6-5-5$ & rkd & 235 & 0.1261 & 0 & 0 & 0.85065 \\
\hline $12-5-5$ & rke & 235 & 0.1049 & 0.0807 & -0.8568 & -0.6739 \\
\hline $12-5-5$ & rkl & 235 & 0.0618 & 0.5534 & 0.9909 & 0.8492 \\
\hline $12-5-10$ & rkg & 235 & 0.0939 & 0.0569 & 0.0200 & 0.8498 \\
\hline $12-5-10$ & rkm & 235 & 0.0249 & 0.9259 & -0.0576 & 0.2339 \\
\hline $12-5-10$ & rkn & 235 & 0.0646 & 0.5844 & -0.6413 & 0.1663 \\
\hline 20-3-3 & rla & 235 & 0.0784 & 0.2255 & 0.2387 & 0.9391 \\
\hline $20-3-3$ & rlb & 235 & 0.0242 & -0.1891 & 0.0751 & 0.6121 \\
\hline $20-3-3$ & rlc & 235 & 0.00711 & 0.0261 & -0.1190 & 0.6494 \\
\hline $20-3-6$ & rle & 235 & 0.0600 & -0.1878 & 0.0084 & 0.5967 \\
\hline $20-3-6$ & rlf & 235 & 0.0289 & -0.0799 & -0.1685 & 0.5787 \\
\hline $20-3-6$ & rlg & 235 & 0.0268 & -0.0733 & -0.1279 & 0.6049 \\
\hline $20-3-9$ & rlh & 235 & 0.0370 & -0.2274 & -0.0450 & 0.5530 \\
\hline $20-3-9$ & rli & 235 & 0.0362 & -0.1763 & -0.0755 & 0.5758 \\
\hline $20-3-9$ & $\mathbf{r l j}$ & 235 & 0.03520 & -0.1312 & 0.2499 & 0.6847 \\
\hline $20-3-9$ & rlk & 235 & 0.03519 & -0.2555 & -0.0034 & 0.5431 \\
\hline $20-3-9$ & rll & 235 & 0.0314 & 0.5758 & 0.1763 & 0.0735 \\
\hline $20-3-9$ & rlm & 235 & 0.0096 & 0.1485 & -0.0353 & 0.7455 \\
\hline 20-3-9 & rln & 235 & 0.0075 & -0.1043 & -0.2231 & 0.5359 \\
\hline $20-3-12$ & rlo & 235 & 0.0292 & -0.2955 & 0.0230 & 0.5148 \\
\hline $20-3-12$ & rlp & 235 & 0.0223 & -0.3105 & 0.0886 & 0.5060 \\
\hline $20-3-12$ & rlq & 235 & 0.0145 & -0.3035 & -0.0363 & 0.4983 \\
\hline $20-3-12$ & rlr & 235 & 0.0137 & -0.2790 & -0.0792 & 0.5065 \\
\hline $20-3-12$ & rls & 235 & 0.00707 & -0.1070 & -0.2526 & 0.5160 \\
\hline $20-3-12$ & rlt & 235 & 0.0037 & -0.1016 & -0.2352 & 0.5292 \\
\hline $20-3-15$ & rlu & 235 & 0.0160 & -0.2548 & -0.0541 & 0.5310 \\
\hline 20-3-18 & rlv & 235 & 0.0321 & -0.2748 & -0.1745 & 0.4771 \\
\hline $20-3-18$ & rlw & 235 & 0.0302 & -0.1473 & -0.2742 & 0.4877 \\
\hline $20-3-18$ & $\mathbf{r l x}$ & 235 & 0.0209 & -0.3497 & -0.0839 & 0.4518 \\
\hline
\end{tabular}

cubic groups, $23(T)$ and $432(O)$, and the icosahedral group $235(I)$. Specifically, the possibilities are:

23: four 3-rings.

432: eight 3-rings or six 4-rings.

235: 20 3-rings, six 5-rings or 12 5-rings.

We now describe these. Maximum girth and optimal coordinates are given for all regular structures in Table 1 and for some stick 2-transitive structures in Table 2.

\subsection{Four 3 -rings}

We find two structures, RCSR symbols rka and rkh, with four 3-rings, catenation symbol 4-3-3 (Fig. 2). In the maximumgirth configuration rka actually has symmetry 432 and is one of just two structures in which the coordinates (apart from a scale factor) are fixed by symmetry (the other is for six 5-rings discussed below). Thus, for unit stick length, $x, y, z=$ $0,1 / \sqrt{6}, 1 / \sqrt{6}$. We note a recent report of molecular assemblies with the rkh structure, wherein it is identified as the 'three-crossed tetrahedral link' (Sawada, Saito et al., 2019). 
Table 2

Data for selected cubic and icosahedral stick 2-transitive links $(c=1, s=2$, $t=1)$.

Coordinates are Cartesian and normalized such that the longer stick length equals 1 . The symbol $x$ indicates that, because of complexity, we did not essay a crossing number.

\begin{tabular}{llllllr}
\hline Symbol & Catenation & Group & Girth & $x$ & $y$ & \multicolumn{1}{c}{$z$} \\
\hline knv & $3-4-2_{5}$ & 23 & 0.0813 & 0.4243 & -0.2645 & \multicolumn{1}{c}{-0.1649} \\
knd & $3-4-0$ & $m \overline{3}$ & 0.25 & 0.5 & 0.25 & \multicolumn{1}{c}{0.0} \\
knq & $6-4-0$ & $m \overline{3}$ & 0.2 & 0.5 & 0.3 & \multicolumn{1}{c}{0.1} \\
kop & $6-4-1_{4}, 4_{2}$ & 432 & 0.1257 & 0.1124 & 0.2227 & -0.5207 \\
kor & $6-4-1_{4}$ & 432 & 0.0369 & 0.1164 & -0.4903 & 0.2549 \\
kos & $6-4-1_{4}, 4_{2}$ & 432 & 0.0218 & 0.2064 & 0.3663 & -0.2750 \\
kot & $6-4-5_{4}$ & 432 & 0.0089 & 0.0471 & 0.4116 & -0.3544 \\
kou & $4-6-3_{4}$ & 432 & 0.0554 & 0.2699 & 0.4209 & 0.3655 \\
kov & $4-6-x$ (trefoil) & 432 & 0.0530 & 0.2967 & 0.0402 & 0.2621 \\
kow & $4-6-x$ (trefoil) & 432 & 0.0381 & 0.3831 & 0.2603 & 0.3755 \\
kox & $4-6-x$ (trefoil) & 432 & 0.0305 & 0.3206 & 0.2986 & 0.5420 \\
koy & $15-4-12_{2}, 2_{0}$ & 235 & 0.0628 & 0.2006 & 0.4580 & 0.3952 \\
koz & $15-4-9_{2}, 3_{4}, 2_{5}$ & 235 & 0.0440 & 0.3836 & 0.3206 & 0.2160 \\
kpm & $10-6-9_{2}$ & 235 & 0.1171 & 0.2908 & 0.4068 & 0.5832 \\
kpn & $10-6-6_{2}, 3_{6}$ & 235 & 0.0643 & 0.1555 & -0.4752 & -0.4991 \\
kpo & $10-6-x$ (trefoil) & 235 & 0.0393 & 0.2174 & 0.4503 & -0.2326 \\
kpp & $6-10-5_{4}$ & 235 & 0.0739 & 0.4665 & -0.1799 & 0.5433 \\
kpq & $6-10-5_{4}$ & 235 & 0.0359 & -0.2705 & -0.4206 & -0.1850 \\
kpr & $6-10-x$ (cinquefoil) & 235 & 0.0811 & -0.3546 & -0.3525 & -0.5529 \\
kps & $6-10-x$ (cinquefoil) & 235 & 0.0725 & 0.2475 & 0.4544 & 0.3953 \\
\hline & & & & & &
\end{tabular}

\subsection{Six 4-rings}

We find two regular structures (rkb and rkc, Fig. 2) in which pairs of 4-rings are parallel and each is catenated to four others, symmetry 432, catenation symbol 6-4-4, crossing number 24 (12 Hopf links). Interestingly, a molecular example of a catenane with the rkc structure has recently been reported (Sawada, Inomata et al., 2019) showing that links with large crossing numbers are realistic targets for synthesis.

\subsection{Eight 3 -rings}

We identify three distinct structures of catenated 3-rings with symmetry 432 (Fig. 2). In every case, pairs of rings are parallel. In one case (rki), each ring is catenated to three others (symbol 8-3-3); in the other two (rkj and rkk) cases,

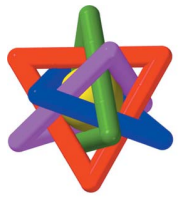

rka 4-3-3

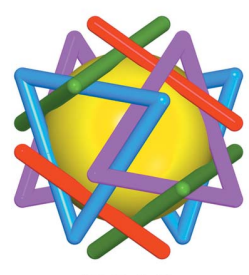

rki 8-3-3

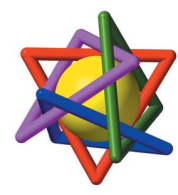

rkh 4-3-3

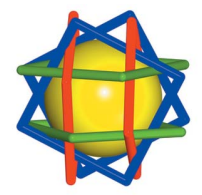

rkb 6-4-4

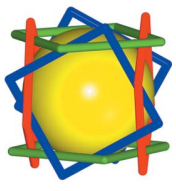

rkc $6-4-4$

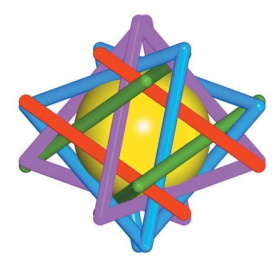

rkj 8-3-6

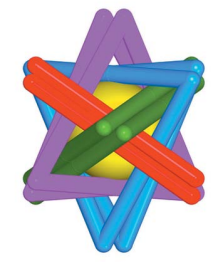

rkk 8-3-6

Figure 2

Regular cubic links. The yellow spheres delineate the internal space.

which are clearly different topologies, each ring is catenated to six others (symbol 8-3-6).

\subsection{Six and 12 5-rings}

There is a unique structure (rkd, Fig. 1) with six linked pentagons with coordinates fixed by symmetry and a scale factor, linking symbol 6-5-5. It is shown again in Fig. 3. For unit stick length the optimum-girth coordinates are $0,0, z$, with $z=\tau / \sqrt{(2+\tau)}=0.8507 \quad[\tau=(1+\sqrt{5}) / 2]$. Note that the corners are at the positions of the vertices of a regular icosidodecahedron (3.5.3.5) with symmetry $m \overline{3} \overline{5}$.

With 12 pentagons, coordinates are now in the general positions of point group 235, and we find five structures. In two of them, each pentagon is linked to five others (symbol 12-5-5); in the other three, each pentagon is linked to ten others (symbol 12-5-10). In Fig. 3 it is clear that these are all topologically distinct (i.e. compare separations of parallel pentagons).

\subsection{Twenty triangles and five tetrahedra}

With 20 linked triangles, in symmetry 235, there are many possibilities. Structures [Figs. 4(a) and 4(b)] have triangles linked to one of three, six, nine, 12,15 or 18 others, and as many as seven local maxima of girth for a given catenation number, so we can be less sure that all reported structures are distinct topologically, although we note that the number of linked structures found (23) is the same as that reported by Lang (2002). In the extreme case there are 180 Hopf links, so a crossing number at least 360 - a daunting number for topological analysis. Accordingly, we report data for, and depict, all 23 observed structures.

The structure map for this system of regular icosahedral links is shown in Fig. 4(c). The coordinates $x^{\prime}$ and $y^{\prime}$ lie on the unit sphere, with $z^{\prime}= \pm \sqrt{1-x^{\prime 2}-y^{\prime 2}}$. The coordinates $x, y, z$, presented in Table 1, are for unit stick length and are related to the coordinates in this plot via $x^{\prime}=x / \sqrt{x^{2}+y^{2}+z^{2}}$ etc. The image intensity depicts $\log$ (girth) for the positive, $z^{\prime} \geq 0$, hemisphere. The black lines decorating the sphere represent paths of zero girth, where sticks intersect. Zones enclosed by
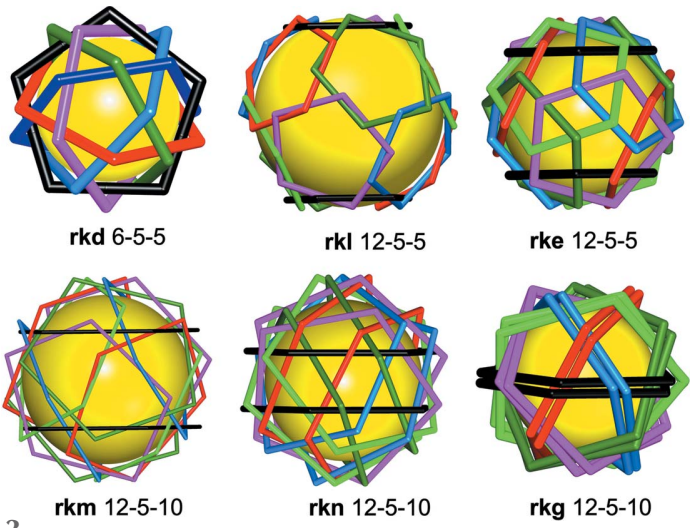

Figure 3

rkn $12-5-10$

rkg $12-5-10$

Regular icosahedral links of catenated pentagons. 


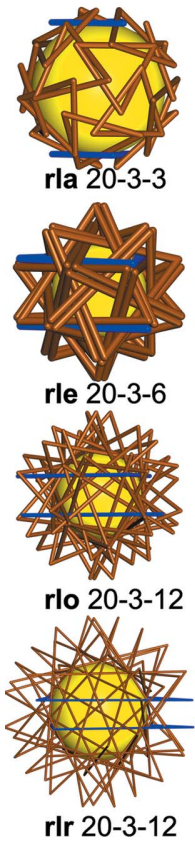

Figure 4
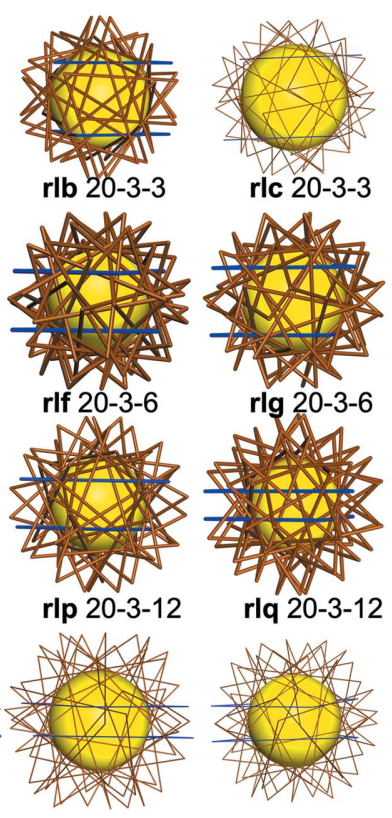

rls 20

(a)

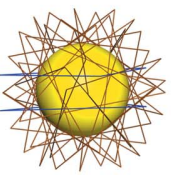

(a) Regular icosahedral links with each loop linked to three, six or 12 others. A pair of parallel triangles is coloured blue. (b) Regular icosahedral links with each loop linked to nine, 15 or 18 others. A pair of parallel triangles is coloured blue.

rlc 20-3-3

rig 20-3-6
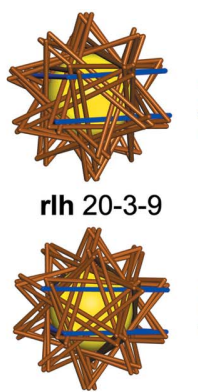

rlk 20-3-9

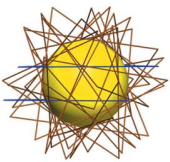

rln 20-3-9

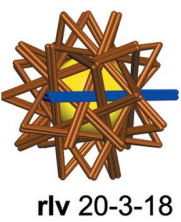

rlt 20-3-12
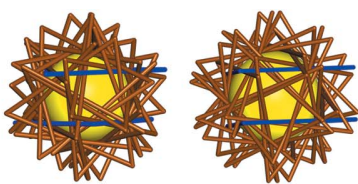

rli 20-3-9
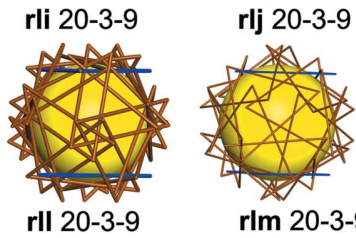

rlm 20-3-9
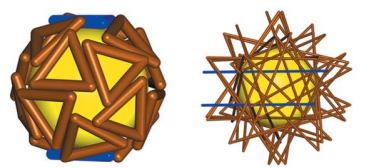

20-3-0

rlu $20-3-15$

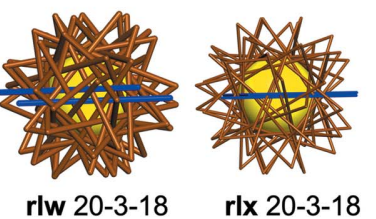

(b)

rlx 20-3-18

the dark lines represent a single structure whose girth varies smoothly within the zone, and is zero at the boundaries. The red dots within each zone indicate the location of the maximum girth for that linked structure (also listed in Table 1). Moving a point $x^{\prime}, y^{\prime}$ across a line means that sticks pass through each other and the link structure changes. When the shared zero-girth boundary is a mirror plane, such as the two dashed lines containing the $\overline{3}$ axis, the neighboring structures are enantiomers. There are 24 structurally distinct zones. Twenty-three are the linked rl* family depicted in Figs. 4( $a$ ) and 4(b) and listed in Table 1. The 24th zone, the clover-leaf pattern at the top right, is where unlinked triangles exist [an example of which, 20-3-0, is shown in Fig. 4(b)]. All 24 zones reside within the spherical triangle depicted by dashed lines which is the asymmetric unit. This asymmetric triangle is repeated 12 times over the full sphere. The symmetry of this structure map is $\overline{3} m$ about the [111] axis shown. This arises because the set of all possible structures includes mirror-

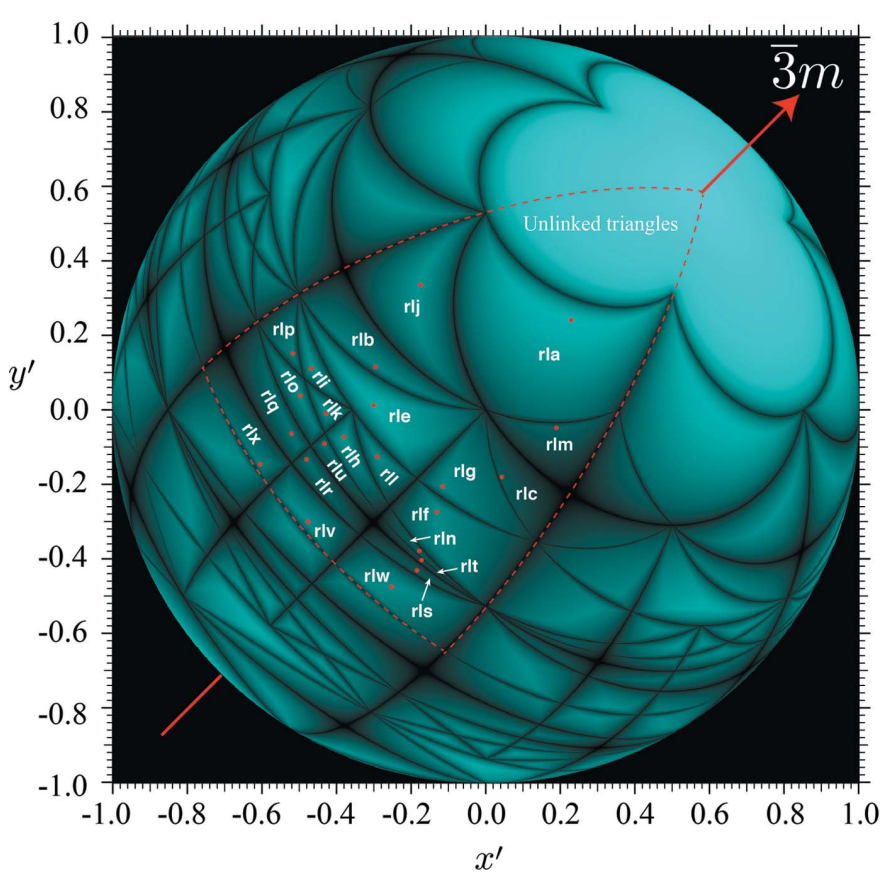

(c)

Figure 4

(c) Structure map on the unit sphere for the regular icosahedral links of catenated triangles depicted in $(a)$ and $(b)$ and listed in Table 1 . The intensity is scaled as $\log$ (girth). Black lines track regions of zero girth. The dashed lines outline an asymmetric unit, of which there are 12 in the point symmetry group $\overline{3} \mathrm{~m}$. The plot confirms that there are 24 structurally distinct zones within each asymmetric unit. Twenty-three are the rl* family. The 24th zone, within the clover-leaf pattern at the top right, is where unlinked triangles exist. Red dots mark the locations of maximum girth for each linked structure. image pairs of each structure and has symmetry $m \overline{3} \overline{5}$. We describe all these structures using just one of the ten possible threefold axes to define a triangle (each structure has ten possible descriptions according to which threefold axis is used to define a triangle), so our structure map has just one-tenth of the symmetry, namely $\overline{3} m$. The lines of zero girth will obey the lowered symmetry. Thus, there are ten available orientations of the symmetry map, depending on which threefold axis we choose to define the system.

In the structure rle [Fig. 4(a)] groups of four triangles come together in a tetrahedral configuration. If groups of three vertices in that configuration are merged, a group of five interlocking tetrahedra (rkz, Fig. 5) results. This structure has transitivity (vertices, edges, faces, polyhedra) 1111 . It is well known to model builders - it occurs as model 24 in the work of Wenninger (1974) - and, we think, it is the only regular grouping of non-intersecting interpenetrating polyhedra. The vertices are at the positions of the vertices of a regular dodecahedron (symmetry $m \overline{3} \overline{5}$ ).

Figure 5
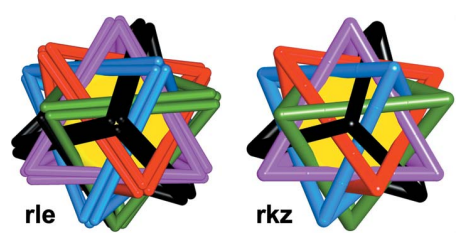

A regular link of 20 triangles (rle) and a related regular cluster of five tetrahedra (rkz). 


\section{Isogonal, stick 2-transitive cubic and icosahedral structures}

First, we ask again: 'Which structures have cubic or icosahedral symmetry?'. Now, a corner has neighbors related by two distinct twofold axes, identifiable by the mutual angle between their axes. The possibilities are:

Three 4-rings, symmetry 23 , axes at $90^{\circ}$.

Six 4-rings, symmetry 432 , axes at $90^{\circ}$.

Four 6-rings, symmetry 432 , axes at $45^{\circ}$.

Fifteen 4-rings, symmetry 235, axes at $90^{\circ}$.

Ten 6-rings, symmetry 235 , axes at $60^{\circ}$.

Six 10-rings, unknotted symmetry 235 , axes at $36^{\circ}$.

Six 10 -rings, knotted symmetry 235 , axes at $72^{\circ}$.

\subsection{Three 4-rings, symmetry 23}

In group 23 there are three mutually perpendicular twofold axes. Two, acting on a vertex, will generate a generally nonplanar quadrangle. We find two distinct ways of forming links. In the first (knv, Fig. 6) pairs of rings are joined as the fivecrossing link, $5_{1}^{2}$, known as the Whitehead link. To indicate this, we extend our notation to include subscripts to the numbers of links, to show the crossing number of the link. So, for this structure, the catenation symbol is $3-4-25$. Note that, for the two-loop Whitehead link (Fig. 6, top right), the two loops are not symmetry equivalent (symmetry 222 , transitivity 24 2). However, the three loops in knv augment the Whitehead link and are now related by symmetry (23) with a simpler transitivity, 121 . This fascinating structure has a large optimum girth $(\sim 0.08)$ and is surely a prime target for synthesis.

There is a second three-loop structure with symmetry 23. In this structure, pairs of loops are not directly linked, and we recognize the structure as Borromean rings (knd) which, as the loops are not directly linked, has optimal symmetry $m \overline{3}$ $\left(T_{h}\right)$. Actually, in this symmetry one can also have six nonlinked quadrangles with transitivity 121 in a structure, knq, which we call 'Borromean twins'. Data (girth, optimal coordinates) for these structures are recorded in Table 2. The Borromean rings have the largest optimum girth (0.25) for any knot or link and it is not surprising that they have

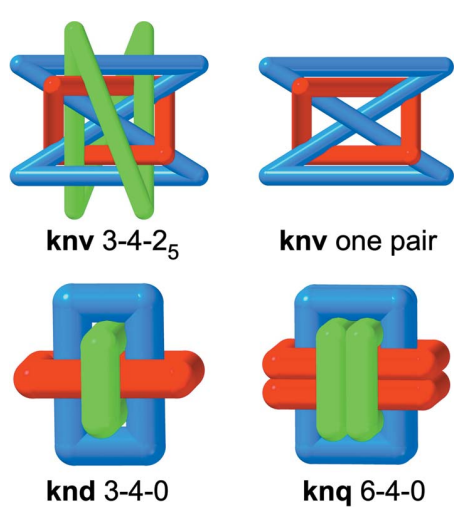

Figure 6 knd 3-4-0

Isogonal cubic links of three quadrangles. Top (knv) quadrangles linked by Whitehead links. Bottom Borromean rings in maximum-symmetry embeddings. Also shown are 'Borromean twins' (six quadrangles).

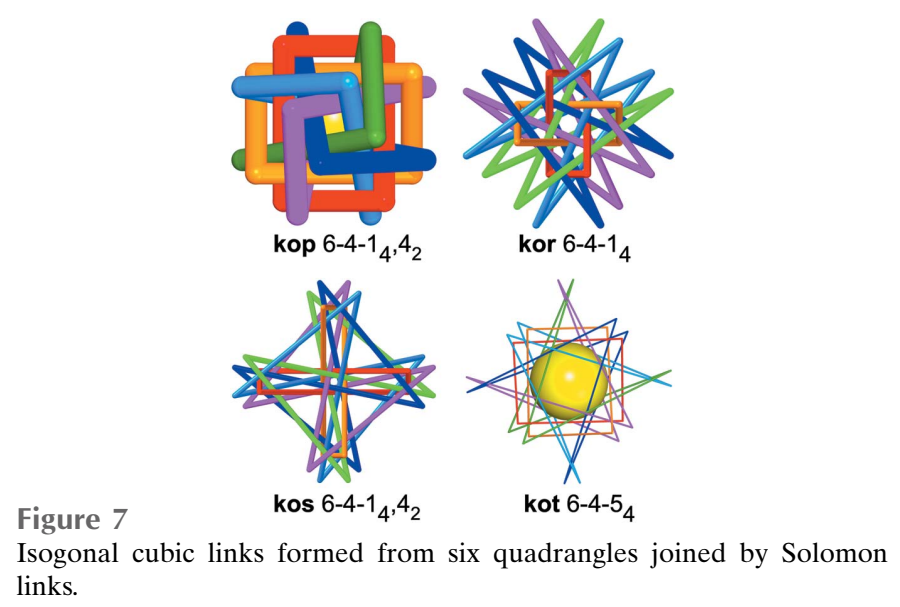

occurred in many molecular assemblies. We believe (see the supporting information) that these two structures are the only isogonal knots or links that have embeddings with mirror symmetry.

\subsection{Six 4-rings, symmetry 432}

In group 432 with neighbors of a vertex produced by twofold axes at $90^{\circ}$, six 4-rings are obtained. Each ring has one other 'opposite' (with a common twofold axis) and four 'adjacent'. The pair of opposite rings form a Solomon (4-crossing) link, as shown in Fig. 7. We find just one largegirth structure $(\mathbf{k o p}$, girth $=0.126)$. In this structure adjacent rings are linked by a Hopf (2-crossing) link so we write the link symbol as $6-4-1_{4}, 4_{2}$. Three other smaller-girth structures, kor, kos and kot, are shown in the figure and data are recorded in Table 2.

\subsection{Four 6-rings, symmetry 432}

In group 432 with neighbors of a vertex produced by twofold axes mutually inclined at $45^{\circ}$, four non-planar 6-rings are obtained. We now find a large number of local maxima of girth in coordinate space and we illustrate just four larger-girth examples. In the first, largest-girth $(\mathbf{k o u}$, girth $=0.055)$ the rings are unknotted skew hexagons and are linked to each other by the 4-crossing Solomon link, as shown in Fig. 8. In our other examples the rings are $3_{1}$ (trefoil) knots linked in distinct ways, as shown in the figure. The complete data set is given as supporting material. We remind the reader that our main purpose is to identify structures amenable to designed synthesis and that large-girth structures are the most favourable in this respect.

\subsection{Fifteen 4-rings, symmetry 235}

Using two twofold axes mutually inclined at $90^{\circ}$ (as usual, to generate the opposite ends of the two stick types), in group 235,15 non-planar quadrangles are generated. These, in turn, form two groups of structures which are fivefold interpenetrations of the three 4-ring structures generated in group 23. These are the Whitehead linked structure, knv, and the Borromean rings knd (Fig. 6). So koy contains five distinct 


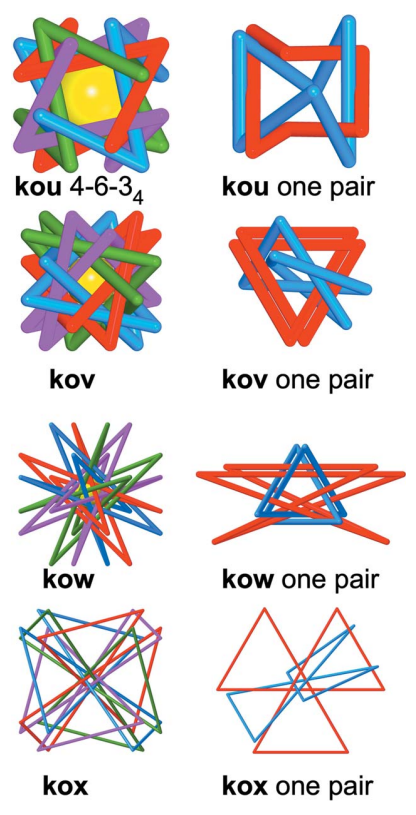

Figure 8

kox

kox one pair

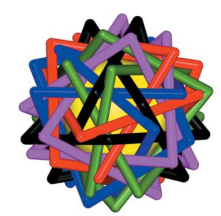

koy $15-4-12_{2}, 2_{0}$

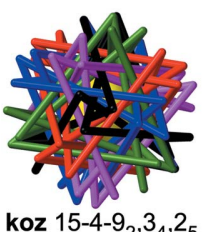

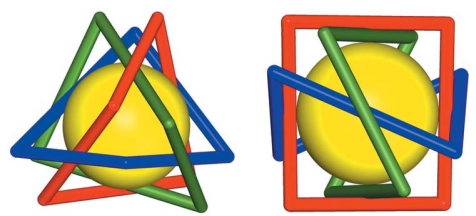

koy one Borromean triplet (knd)
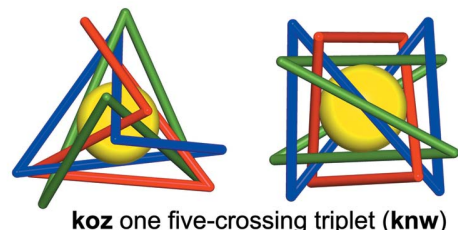

Figure 9

Isogonal icosahedral links of 15 rectangles comprised of five interpenetrating Borromean triplets (top) and five interpenetrating triplets of Whitehead links (bottom).

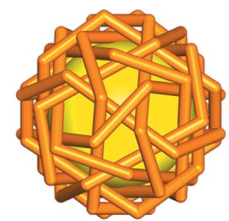

kpm 10-6 -9,

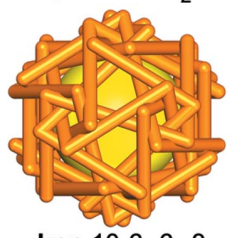

kpn $10-6-6_{2}, 3_{6}$

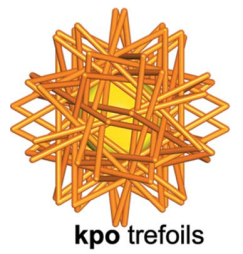

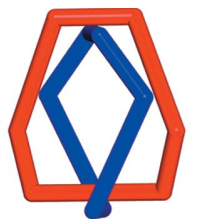

kpm one pair

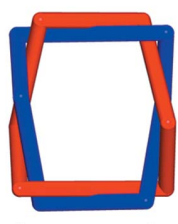

kpn one pair

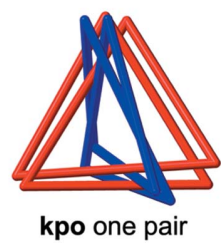

Figure 10

Isogonal icosahedral links of ten hexagons showing different patterns of catenation.
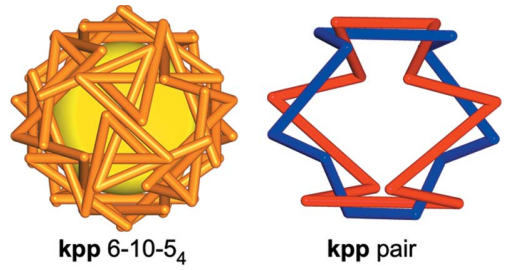

ated. We show two larger-girth examples, kpp and kpq, in Fig. 11. In both, each ring is catenated to five others by a Solomon link - catenation symbol 6-10-54.

If instead, the chosen twofold axes are mutually inclined at $72^{\circ}$, then knotted decagons are generated which are cinquefoil knots [torus knot $(5,2)$ discussed below]. In Fig. 12 we show two larger-girth examples, kpr and kps. We do not essay a linking symbol, but note that each linked pair has a crossing number of 10 just from self-crossings.

Figure 11
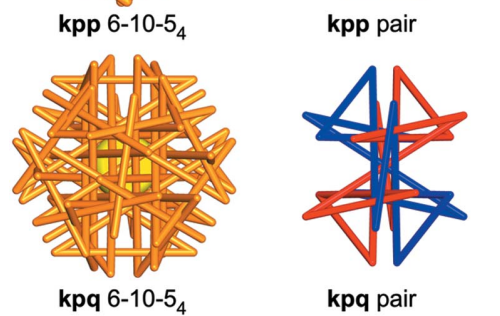

kpq pair

Isogonal icosahedral links of six unknotted decagons. 


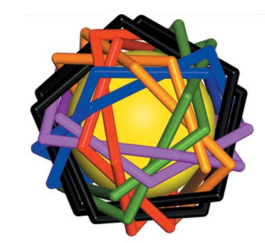

kpr

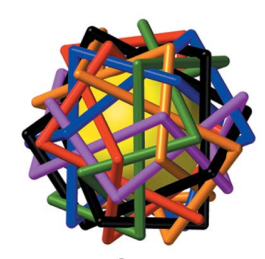

kps

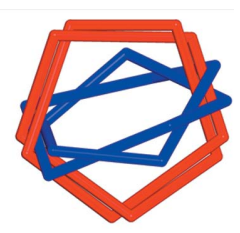

kpr one pair

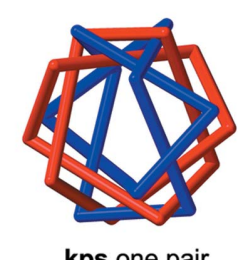

kps one pair

Figure 12

Isogonal icosahedral links of six knotted (cinquefoil) decagons.

\section{Knots and links with axial symmetry}

There are infinitely many knots and links with isogonal piecewise linear embeddings. They have the symmetries of the axial groups $n 2\left(D_{n}\right)$ with $n$ odd or $n 22\left(D_{n}\right)$ with $n$ even. As mentioned earlier, they can be drawn on the surface of a torus with non-intersecting threads, hence the name torus knots and links.

They are characterized by two integers $(p, q)$ with $p \geq q \geq 2$. If $p, q$ are coprime the structure is a knot, otherwise it is a link with the number of loops equal to the greatest common divisor, $r$, of $p$ and $q$. Specifically, there are $r$ interwoven $(p / r, q / r)$ knots. In this context note that $(p, 1)$ is an unknotted $2 p$-gon.

To get an embedding, we use Cartesian coordinates and note that with symmetry $n 2$ or $n 22$ and the $n$-fold axis along $z$, the set of $2 n$ points derived from one point $\mathbf{x}=(x, y, z)$ when projected down $z$, lie on a circle with points with positive and negative $z$ coordinates alternating. Without loss of generality, we can take one twofold axis to be along the $x$ axis and the point, $\mathbf{x}$, to be the positive triplet closest to the $x$ axis (see Fig. 13). To generate the knot/link we now add sticks from $\mathbf{x}$ to points that are the $i$ th neighbor clockwise and $j$ th neighbor anticlockwise, as shown by the blue lines in the figure. To make a knot/link, $i$ and $j$ must be odd. $q$ in the knot symbol is $q=(i+j) / 2$. Note that $q$ defines the structure for a given $p$; for example, $i, j=1,5$ and 3,3 are topologically the same.

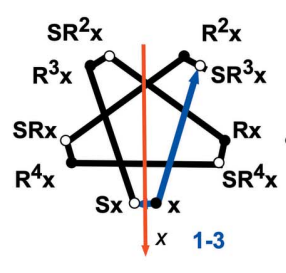

$(5,2)$

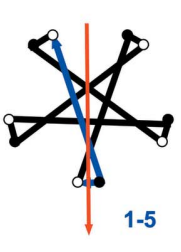

$1-5$

$$
(5,3)
$$

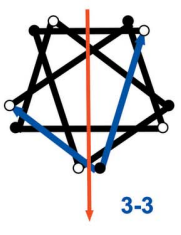

$(5,3)$

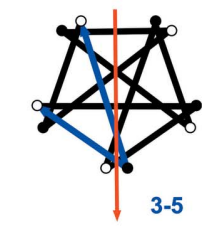

$(5,4)$
Figure 13

Generation of the torus knots $(5, q)$. The fivefold axis is normal to the page and a twofold axis is along the $x$ direction (red arrow). The points are generated by the fivefold rotation matrix $\mathbf{R}$ and the twofold rotation matrix $\mathbf{S}$. Numbers in blue are the number of steps made by the blue arrows; $q$ is half the sum of those numbers.

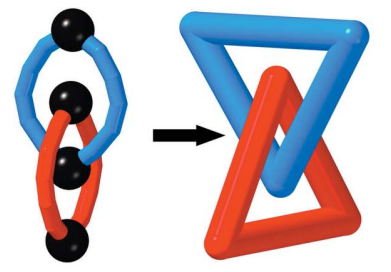

$(2,2)$ 2-3-1

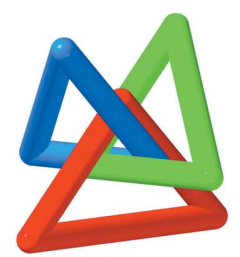

$(3,3) 3-3-2$

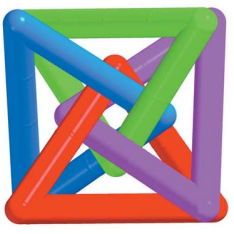

$(4,4) 4-3-3$
Figure 14

Torus links $(p, p)$. On the left $(2,2)$ is shown as linked digons and piecewise linear as linked triangles. The symmetries are 222, 32 and 422, respectively, and the transitivity 221 in each case.

The structures can be derived from a segment of a linear bundle of $q$ helices repeating $p$ times with opposite ends of threads joined. The crossing number of the knot/link is $p(q-1)$.

Torus knots are particularly important in natural DNA structures (e.g. Reith et al., 2012). They also feature prominently in the knots most amenable to construction by molecular self-assembly (Marenda et al., 2018). Notable recently achieved molecular examples are (4,3) (Danon et al., 2017), $(8,3)$ (Kim et al., 2018), and (7,2) and (8,2) (Inomata et al., 2020).

The links with $p=q$ are usually included in the family but the loops are digons, which are not realizable piecewise linear. We note that $(2,2)$ is just the Hopf link $2_{1}^{2}$ and $(3,3)$ is $6_{3}^{3}$ - both with piecewise linear embeddings with triangles and transitivity 22 , as shown in Fig. 14.

We have determined optimum-girth configurations for all knots and links $(p, q)$ for $p \leq 13$. Figs. 15 and 16 illustrate knots for $p \leq 11$ and Figs. 17 and 18 illustrate links for $p \leq 12$.

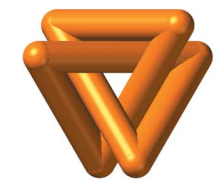

$(3,2)$

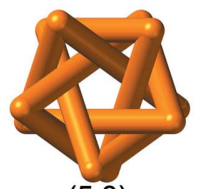

$(5,3)$

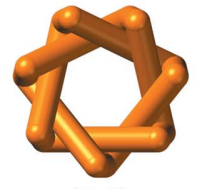

$(7,2)$

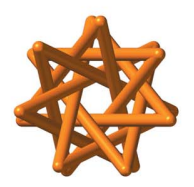

$(7,5)$

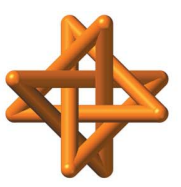

$(4,3)$

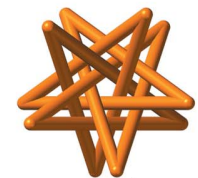

$(5,4)$

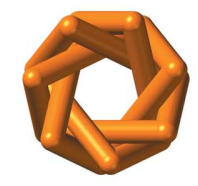

$(7,3)$

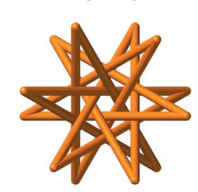

$(7,6)$

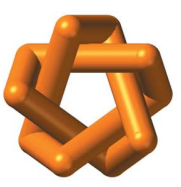

$(5,2)$

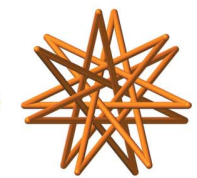

$(6,5)$

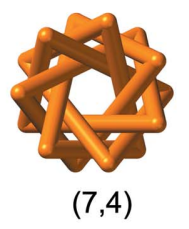

$(7,4)$

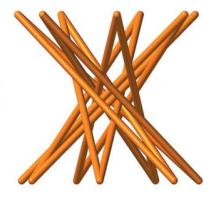

$(7,6)$
Figure 15

Maximum-girth embeddings for torus knots $(p, q)$ for $p=3-7$. 
Table 3

Data for torus knots $(p, q)$ for $p \leq 10$ and links $(p, q) p \leq 12$ (marked $*$ ).

For these knots, $(c=1, s=2, t=1)$. Cartesian coordinates are for a twofold axis along the $x$ direction, and longer stick length equal to 1.0.

\begin{tabular}{|c|c|c|c|c|c|c|}
\hline Symbol & Girth & $x$ & $y$ & $z$ & Stick 2 & $\begin{array}{l}\text { Angle } \\
\left({ }^{\circ}\right)\end{array}$ \\
\hline$(3,2)$ & 0.1748 & 0.1354 & 0.4363 & 0.2442 & 0.8297 & 50.19 \\
\hline$(4,2)^{*}$ & 0.2051 & 0.4225 & 0.2175 & 0.2673 & 0.6892 & 65.50 \\
\hline$(4,3)$ & 0.0977 & 0.0955 & 0.4017 & 0.2978 & 0.9214 & 35.08 \\
\hline$(5,2)$ & 0.2057 & 0.2800 & -0.4197 & -0.2718 & 0.6083 & 68.34 \\
\hline$(5,3)$ & 0.1351 & 0.1691 & 0.4002 & 0.2997 & 0.8265 & 52.77 \\
\hline$(5,4)$ & 0.0606 & 0.0718 & 0.3836 & 0.3207 & 0.9531 & 27.04 \\
\hline$(6,2)^{*}$ & 0.1981 & 0.3318 & -0.4203 & -0.2708 & 0.5632 & 66.07 \\
\hline$(6,3)$ & 0.1492 & 0.2289 & 0.4011 & 0.2986 & 0.7525 & 61.71 \\
\hline$(6 ; 4) *$ & 0.0915 & 0.1326 & 0.3857 & 0.3181 & 0.8852 & 43.49 \\
\hline$(6,5)$ & 0.0412 & 0.0576 & 0.3741 & 0.3317 & 0.9685 & 22.10 \\
\hline$(7,2)$ & 0.1882 & 0.3767 & -0.4221 & -0.2679 & 0.5395 & 61.89 \\
\hline$(7,3)$ & 0.1535 & 0.2799 & -0.4029 & -0.2961 & 0.6964 & 65.85 \\
\hline$(7,4)$ & 0.1072 & 0.1847 & 0.3884 & 0.3149 & 0.8251 & 53.67 \\
\hline$(7,5)$ & 0.0657 & 0.1088 & 0.3770 & 0.3285 & 0.9173 & 37.01 \\
\hline$(7,6)$ & 0.0299 & 0.0483 & 0.3685 & 0.3379 & 0.9773 & 18.73 \\
\hline$(8,2)^{*}$ & 0.1781 & 0.4164 & -0.4244 & -0.2643 & 0.5287 & 57.23 \\
\hline$(8,3)$ & 0.1532 & 0.3248 & -0.4052 & -0.2929 & 0.6537 & 67.20 \\
\hline$(8,4)^{*}$ & 0.1152 & 0.2306 & 0.3912 & 0.3114 & 0.7750 & 59.96 \\
\hline$(8,5)$ & 0.0800 & 0.1539 & 0.3800 & 0.3250 & 0.8680 & 47.20 \\
\hline$(8,6) *$ & 0.0494 & 0.0924 & 0.3714 & 0.3348 & 0.9373 & 32.24 \\
\hline$(8,7)$ & 0.0227 & 0.0417 & 0.3649 & 0.3418 & 0.9829 & 16.27 \\
\hline$(9,2)$ & 0.1685 & 0.4523 & -0.4268 & -0.2604 & 0.5259 & 52.70 \\
\hline$(9,3) *$ & 0.1506 & 0.3651 & -0.4076 & -0.2896 & 0.6213 & 66.89 \\
\hline$(9,4)$ & 0.1188 & 0.2717 & -0.3939 & -0.3079 & 0.7334 & 63.72 \\
\hline$(9,5)$ & 0.0886 & 0.1944 & 0.3830 & 0.3214 & 0.8243 & 54.23 \\
\hline$(9,6) *$ & 0.0619 & 0.1320 & 0.3743 & 0.3315 & 0.8961 & 42.07 \\
\hline$(9,7)$ & 0.0385 & 0.0804 & 0.3675 & 0.3390 & 0.9507 & 28.57 \\
\hline$(9,8)$ & 0.0178 & 0.0366 & 0.3625 & 0.3444 & 0.9866 & 14.39 \\
\hline$(10,2) *$ & 0.1596 & 0.4852 & -0.4292 & -0.2566 & 0.5279 & 48.56 \\
\hline$(10,3)$ & 0.1469 & 0.4019 & -0.4100 & -0.2862 & 0.5965 & 65.60 \\
\hline$(10,4) *$ & 0.1200 & 0.3092 & -0.3967 & -0.3044 & 0.6988 & 65.79 \\
\hline$(10,5)$ & 0.0936 & 0.2313 & 0.3859 & 0.3179 & 0.7864 & 59.06 \\
\hline$(10,6) *$ & 0.0701 & 0.1679 & 0.3772 & 0.3282 & 0.8580 & 49.29 \\
\hline$(10,7)$ & 0.0493 & 0.1156 & 0.3703 & 0.3360 & 0.9159 & 37.93 \\
\hline$(10,8) *$ & 0.0309 & 0.0713 & 0.3648 & 0.3419 & 0.9602 & 25.66 \\
\hline$(10,9)$ & 0.0144 & 0.0327 & 0.3608 & 0.3462 & 0.9892 & 12.91 \\
\hline$(12,2)^{*}$ & 0.1439 & 0.5438 & -0.4335 & -0.2491 & 0.5396 & 41.56 \\
\hline$(12,3) *$ & 0.1384 & 0.4673 & -0.4146 & -0.2795 & 0.5639 & 61.5 \\
\hline$(12,4) *$ & 0.1184 & 0.3759 & -0.4017 & -0.2977 & 0.6455 & 66.80 \\
\hline$(12,6) *$ & 0.0788 & 0.2319 & 0.3827 & 0.3217 & 0.7932 & 58.53 \\
\hline$(12,8)^{*}$ & 0.0470 & 0.1323 & 0.3698 & 0.3365 & 0.9010 & 41.53 \\
\hline$(12,9) *$ & 0.0335 & 0.0930 & 0.3651 & 0.3416 & 0.9415 & 31.67 \\
\hline$(12,10) *$ & 0.0212 & 0.0582 & 0.3613 & 0.3456 & 0.9725 & 21.33 \\
\hline
\end{tabular}

Data (girth, coordinates and stick lengths) are reported in Table 3. The rest of the structures are reported in the supporting information.

Optimum girth in the torus knots and links is always less than $\frac{1}{4}$ and it generally decreases with increasing $p$ and $q$. For example, a plot of girth versus $q$ for the $(13, q)$ family (Fig. 19, blue squares) shows that girth decreases monotonically with $q$, with the peak value occurring at $q=2$. Because $p=13$ is a prime number, all of that family are knots (see the supporting information). The red circles in the plot indicate how girth for the $(p, 2)$ structures varies with $p$. In this case, there is a peak girth $g=0.2357$ for $p=4$, and the girth then decreases monotonically with $p$. For $p \geq 7$, girth values fit well to a negative exponential,

$$
g(p) \simeq 0.0525+0.2362 \exp (-0.0790 p)
$$
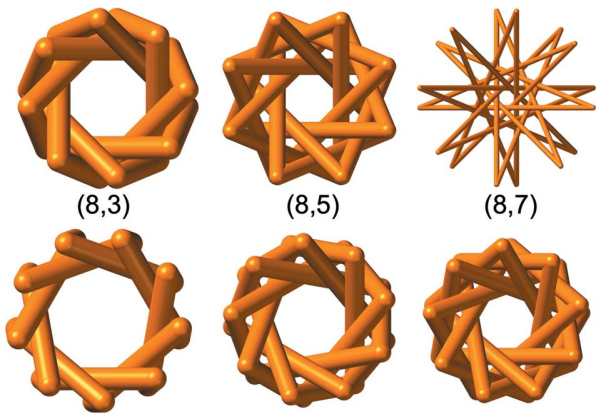

$(9,4)$
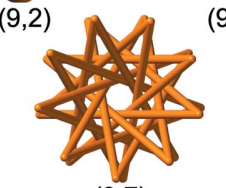

$(9,7)$

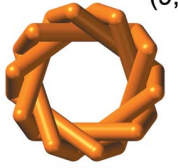

$(10,3)$

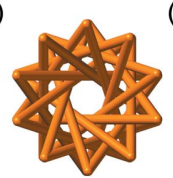

$(10,7)$
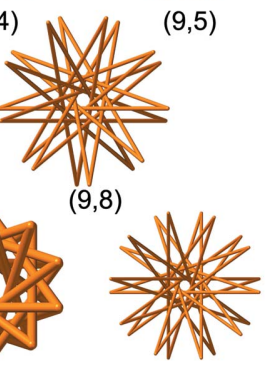

$(10,9)$
Figure 16

Maximum-girth embeddings for torus knots $(p, q)$ for $p=8-10$.

It is notable in the graph (Fig. 19) that the maximum girth for $(3,2)$ (Fig. 15, top left) is less than that for $(4,2)$ (Fig. 17, top left), breaking the monotonic trend. It appears that the tighter angles in (3,2), compared with (4.2), constrict the stick widths, despite $(3,2)$ appearing to have the larger girth in the projection shown along $z$.

\section{Concluding remarks}

According to Grünbaum \& Shephard (1985) there are no knots with cubic or icosahedral symmetry. The symmetry then has to be one of the uniaxial groups. They state further that there are no knots with mirror planes normal to that axis other than $2 / m$. However, they considered knots to be composite (a

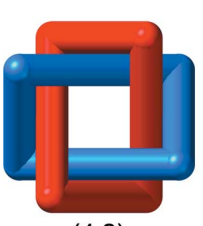

$(4,2)$

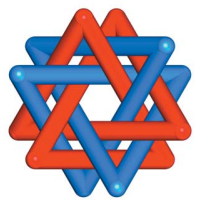

$(6,4)$

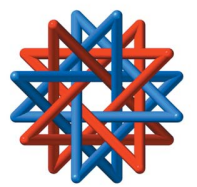

$(8,6)$
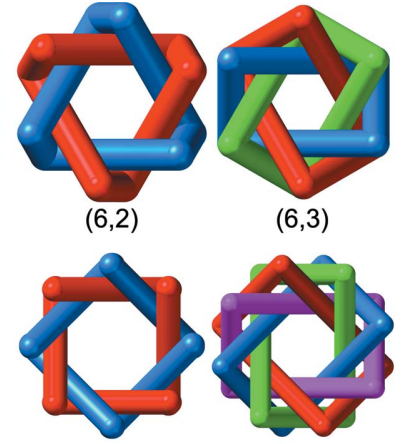

$(8,2)$

$(8,4)$

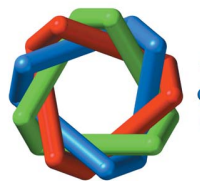

$(9,3)$

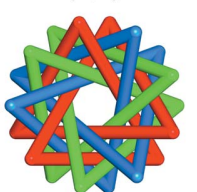

$(9,6)$
Figure 17

Maximum-girth embeddings for torus links $(p, q)$ for $p=1-9$. 


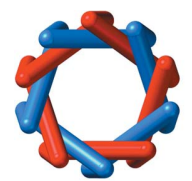

$(10,2)$

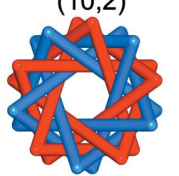

$(10,6)$

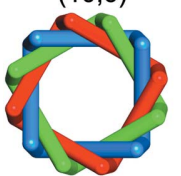

$(12,3)$

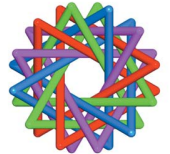

$(12,8)$

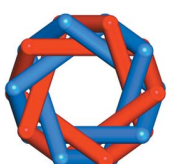

$(10,4)$

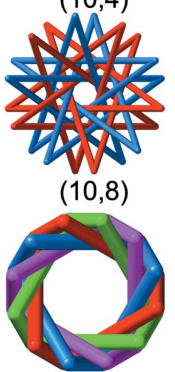

$(12,4)$

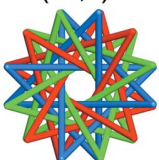

$(12,9)$

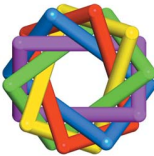

$(10,5)$

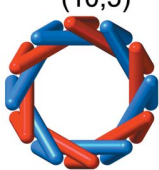

$(12,2)$

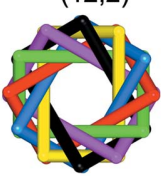

$(12,6)$

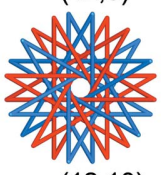

$(12,10)$
Figure 18

Maximum-girth embeddings for torus links $(p, q)$ for $p=10-12$.

linking of two or more smaller knots) as well as prime (not composite).

On the symmetry of embeddings of knots and links we offer the following educated guesses ('conjectures' is too grand a word in this case). Always excepting Borromean rings, which are sui generis, for finite (no translational symmetry) prime knots and links:

(i) All isogonal embeddings are chiral.

(ii) The only stick-transitive (isotoxal) structures are the links we have described here (Section 2).

(iii) No prime knots have mirror symmetry, so the only achiral embeddings of these have symmetry $\bar{N}$, where $N$ is odd or a multiple of 4 .

In this paper we have restricted the discussion to knots and links - structures in which two sticks meet at a corner. However, molecular structures are also found that are based on tangled polyhedra (e.g. Domoto et al., 2020), entering the

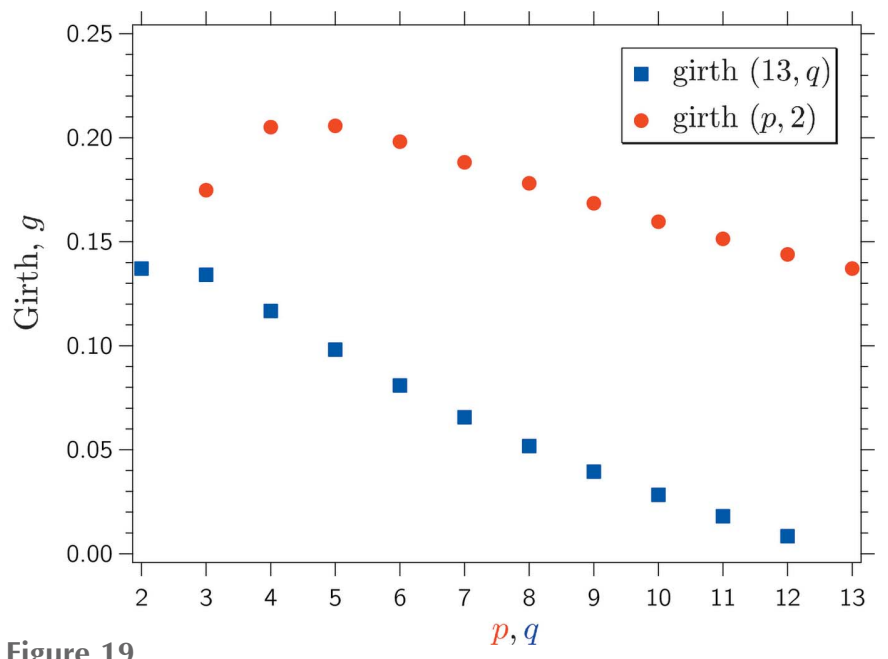

Plot showing the girths $(p, 2)$ and $(13, q)$ for torus knots.
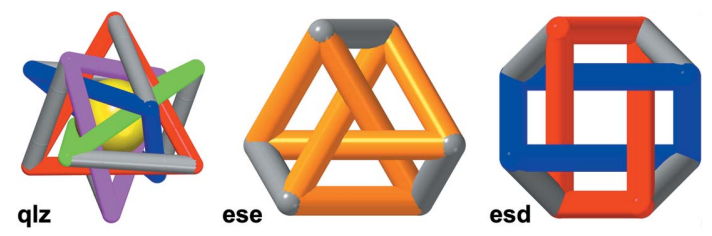

Figure 20

Examples of 3-c isogonal structures formed by adding extra edges (grey in the figure) to isogonal 2-c structures.

more general mathematical world of ravels (Castle et al., 2008, 2009). In a piecewise linear embedding there are now threeand higher-valent vertices. Isogonal three-valent structures can readily be derived from the structures presented here. We adduce three simple examples (Fig. 20). From the [4] catenane rka (Fig. 2) adding an extra edge produces the tangled truncated tetrahedron $3.6^{2}$ (qlz) shown (symmetry now 23). From the trefoil knot $(3,2)$ adding an extra link produces the complete bipartite graph $K_{3,3}$. This occurs in molecular structures as a 'Möbius ladder' (Flapan, 2015). Finally, adding extra edges to the Solomon link $(4,2)$ produces the tangled cube, esd, the 'wreath cube' (Hyde \& Schröder-Turk, 2007). For amplification see the supporting information.

\section{Acknowledgements}

We are grateful to Marjorie Senechal for alerting us to Orderly Tangles (Holden, 1983) and related work; to Davide Proserpio for assistance in acquiring hard-to-get publications; and to David Palmer for tweaks to CrystalMaker which enabled easy import and visualization of structural data. All structure illustrations were made with CrystalMaker.

\section{References}

Adams, C. C. (1994). The Knot Book. Providence: American Mathematical Society.

Bonneau, C. \& O'Keeffe, M. (2015). Acta Cryst. A71, 82-91.

Bruns, C. J. \& Stoddart, J. F. (2016). The Nature of the Mechanical Bond: From Molecules to Machines. New York: Wiley.

Castle, T., Evans, M. E. \& Hyde, S. T. (2008). New J. Chem. 32, 14841492.

Castle, T., Evans, M. E. \& Hyde, S. T. (2009). New J. Chem. 33, $2107-$ 2133.

Danon, J. J., Krüger, A., Leigh, D. A., Lemonnier, J. F., Stephens, A. J., Vitorica-Yrezabal, I. J. \& Woltering, S. L. (2017). Science, 355, 159162.

Domoto, Y., Abe, M., Kikuchi, T. \& Fujita, M. (2020). Angew. Chem. Int. Ed. 59, 3450-3454.

Fielden, S. D. P., Leigh, D. A. \& Woltering, S. L. (2017). Angew. Chem. Int. Ed. 56, 11166-11194.

Flapan, E. (1987). Pac. J. Math. 129, 57-66.

Flapan, E. (1988). Discrete Appl. Math. 19, 157-166.

Flapan, E. (2015). Knots, Molecules and the Universe. Providence: American Mathematical Society.

Grünbaum, B. \& Shephard, G. C. (1985). Math. Mag. 58, 161-165.

Holder, A. (1983). Orderly Tangles. New York: Columbia University Press.

Horner, K. E., Miller, M. A., Steed, J. W. \& Sutcliffe, P. M. (2016). Chem. Soc. Rev. 45, 6432-6448.

Hoste, J., Thistlethwaite, M. \& Weeks, J. (1998). Math. Intelligencer, 20, 33-48. 
Hu, G., Zhai, X.-D., Lu, D. \& Qiu, W.-Y. (2009). J. Math. Chem. 46, 592-603.

Hyde, S. T. \& Schröder-Turk, G. E. (2007). Acta Cryst. A63, 186197.

Inomata, Y., Sawada, T. \& Fujita, M. (2020). Chem, 6, 294-303.

Jablan, S., Radović, L. \& Sazdanović, R. (2011). Bridges, paper 14. http://bridgesmathart.org/2011/cdrom/proceedings/14/paper_14.pdf.

Kim, D. H., Singh, N., Oh, J., Kim, E.-H., Jung, J., Kim, H. \& Chi, K.-W. (2018). Angew. Chem. Int. Ed. 57, 5669-5673.

Lang, R. J. (2002). Origami ${ }^{3}$, edited by T. Hull, pp. 153-168. Natick: A. K. Peters.

Leigh, D. A., Pirvu, L. \& Schaufelberger, F. (2019). J. Am. Chem. Soc. 141, 6054-6059.

Li, M., Li, D., O'Keeffe, M. \& Yaghi, O. M. (2014). Chem. Rev. 114, 1343-1370.

Lim, C. H. \& Jackson, S. E. (2015). J. Phys. Condens. Matter, 27, 354101.

Liu, Y., O'Keeffe, M., Treacy, M. M. J. \& Yaghi, O. M. (2018). Chem. Soc. Rev. 47, 4642-4664.

Marenda, M., Orlandini, E. \& Micheletti, C. (2018). Nat. Commun. 9, 3051.

O'Keeffe, M., Peskov, M. A., Ramsden, S. J. \& Yaghi, O. M. (2008). Acc. Chem. Res. 41, 1782-1789.
O'Keeffe, M. \& Treacy, M. M. J. (2020). Acta Cryst. A76, 110-120.

Pieters, B. J., van Eldijk, M. B., Nolte, R. J. \& Mecinović, J. (2016). Chem. Soc. Rev. 45, 24-39.

Reith, D., Cifra, P., Stasiak, A. \& Virnau, P. (2012). Nucleic Acids Res. 40, 5129-5137.

Sawada, T., Inomata, Y., Shimokawa, K. \& Fujita, M. (2019). Nat. Commun. 10, 5687.

Sawada, T., Saito, A., Tamiya, K., Shimokawa, K., Hisada, Y. \& Fujita, M. (2019). Nat. Commun. 10, 921.

Stasiak, A., Katritch, V. \& Kauffman, L. H. (1998). Ideal Knots, Vol. 19. Singapore: World Scientific.

Thompson, B. \& Hyde, S. T. (2018). Isr. J. Chem. 58, 1144-1156.

Tranchemontagne, D., Ni, Z., O'Keeffe, M. \& Yaghi, O. M. (2008). Angew. Chem. Int. Ed. 47, 5136-5147.

Wenninger, M. (1974). Polyhedron Models. Cambridge University Press.

Wu, Q., Rauscher, P. M., Lang, X., Wojtecki, R. J., de Pablo, J. J., Hore, M. J. A. \& Rowan, S. J. (2017). Science, 358, 1434-1439.

Yaghi, O. M., O'Keeffe, M., Ockwig, N. W., Chae, H. K., Eddaoudi, M. \& Kim, J. (2003). Nature, 423, 705-714.

Zhang, L., Stephens, A. J., Nussbaumer, A. L., Lemonnier, J. F., Jurček, P., Vitorica-Yrezabal, I. J. \& Leigh, D. A. (2018). Nat. Chem. 10, 1083-1088. 